\title{
A New Paradigm of Technology-Enabled 'Vital Signs' for Early Detection of Health Change for Older Adults
}

\author{
Marilyn J. Rantz ${ }^{a, d}$ Marjorie Skubic ${ }^{b}$ Mihail Popescu ${ }^{c}$ Colleen Galambos ${ }^{e}$ \\ Richelle J. Koopman ${ }^{d}$ Gregory L. Alexander ${ }^{a}$ Lorraine J. Phillips ${ }^{a}$ \\ Katy Musterman ${ }^{f}$ Jessica Back ${ }^{f}$ Steven J. Miller ${ }^{a}$ \\ ${ }^{a}$ Sinclair School of Nursing, ${ }^{b}$ Electrical and Computer Engineering, ${ }^{c}$ Department of Health Management and \\ Informatics and d Curtis W. and Ann H. Long Department of Family and Community Medicine, School of Medicine, \\ eSchool of Social Work, and ${ }^{\mathrm{f}}$ TigerPlace, Sinclair School of Nursing, University of Missouri, Columbia, Mo., USA
}

\section{Key Words}

Technology $\cdot$ Vital signs $\cdot$ Older adults $\cdot$ Sensors

\begin{abstract}
Environmentally embedded (nonwearable) sensor technology is in continuous use in elder housing to monitor a new set of 'vital signs' that continuously measure the functional status of older adults, detect potential changes in health or functional status, and alert healthcare providers for early recognition and treatment of those changes. Older adult participants' respiration, pulse, and restlessness are monitored as they sleep. Gait speed, stride length, and stride time are calculated daily, and automatically assess for increasing fall risk. Activity levels are summarized and graphically displayed for easy interpretation. Falls are detected when they occur and alerts are sent immediately to healthcare providers, so time to rescue may be reduced. Automated health alerts are sent to healthcare staff, based on continuously running algorithms applied to the sensor data, days and weeks before typical signs or symptoms are detected by the person, family members, or healthcare providers. Discovering these new functional status 'vital signs', developing auto-
\end{abstract}

mated methods for interpreting them, and alerting others when changes occur have the potential to transform chronic illness management and facilitate aging in place through the end of life. Key findings of research in progress at the University of Missouri are discussed in this viewpoint article, as well as obstacles to widespread adoption.

(c) 2014 S. Karger AG, Basel

How does a person detect that 'I am not feeling quite right', essentially that a change in health is about to occur? Below is a description of a typical scenario for families of older people.

Mary, 86 years of age, lives alone; she has a history of well-controlled hypertension, adult-onset diabetes, and osteoarthritis of both knees. She drives to church a few miles from her home twice weekly for activities and plays bridge weekly for serious but lively card games. Her daughter checks by phone daily and accompanies her to primary care appointments routinely scheduled twice yearly. Awaking earlier than usual this morning, while walking to the bathroom she experienced slight dizziness and fell - luckily there were no obstacles so she was not injured. After using the bathroom, she felt tired, a bit thirsty, but not hungry, so she decided to nap in

\section{KARGER 125}

(C) 2014 S. Karger AG, Basel

0304-324X/14/0613-0281\$39.50/0

E-Mail karger@karger.com

www.karger.com/ger
Marilyn J. Rantz, PhD, RN, FAAN

S406 Sinclair School of Nursing, University of Missouri

Columbia, MO 65211 (USA)

E-Mail RantzM@missouri.edu 
her favorite chair. She awakened to the phone later in the morning, and her daughter, Louise, was alarmed that her mom was napping, especially after asking what she had for breakfast and learning she had not eaten and was not hungry. Mary tried to reassure Louise that nothing was wrong; she did not mention falling or dizziness, thinking it was not relevant. Later, after work, Louise decided to drop in to check on her mom. It took an unusually long time for Mary to answer her door, she was still in her pajamas; Louise noticed an afghan tossed in her recliner chair where she had apparently spent the day. Mary's gait was unsteady as she walked from the door toward the chair. Louise asked how her day had been and Mary confirmed she was just really tired so she had dozed the day away in her chair. Louise looked about and saw no evidence of her mom having eaten and her usual tea cup was not nearby... .

Subtle changes in usual patterns of activities of daily living, such as bathroom activities, sleep patterns, eating, drinking, gait, falls, and other behaviors or physical measures can be early indicators of health changes for older adults. The challenge for healthcare providers, families, and the older person is detecting and measuring subtle changes so that people can benefit from early illness recognition without overwhelming them with unnecessary vigilance that can negatively affect quality of life and independence. Subtle changes in intermittent vital signs (pulse and respiration rates, blood pressure, weight, pulse oximetry, or blood sugar measurement) taken daily, weekly, or just at times of primary care office visits, are often not significant enough to trigger detection of early health problems.

This challenge, identifying a new paradigm of vital signs that are technology enabled, is one that our Eldertech Research team at the University of Missouri undertook a dozen years ago. Healthcare members of the team challenged engineers: 'Give us new technology to help us find novel solutions to the persistent problems experienced by older adults' [1]. Together, this work has taken several paths, but all toward the same goal: helping older adults maintain and maximize health and independence, allowing them to age in the place where they want to live through the end of their lives. With newly developed technological methods to detect subtle changes, early interventions can be more effective in restoring health, avoiding hospitalization in many cases, and helping people stay independent as long as possible [2-4].

The purpose of this viewpoint is threefold: (1) discuss research behind the technology-enabled 'vital signs' for early detection of health change that our Eldertech Research team has conducted, (2) discuss clinical implications for mainstream adoption and use of these vital signs for early interventions to help older adults, their families, and healthcare providers, and (3) present some obstacles to overcome for mainstream adoption.

\section{Why Early Detection of Health Change?}

Problems in chronic disease management often cause loss of independence for aging Americans. In 2012, 1 in 2 Americans (117 million) had at least one chronic condition and $26 \%$ had multiple conditions [5]. Moreover, $84 \%$ of healthcare costs in 2006 were for the $50 \%$ of the population with a chronic condition [6]. Chronic diseases especially affect older adults [7] in whom exacerbations result in dramatic decline/changes in health and functional status, hospitalization, complex treatment interventions, and high cost [8]. Early illness recognition and early treatment are keys to improving health status after exacerbation of a chronic or acute illness $[9,10]$. Recognition of small changes in health conditions are essential for early interventions when treatment is most effective, prevention of dramatic decline is still possible, and costs can be controlled.

Functional decline often heralds episodes of acute illness or exacerbation of chronic illness [11-13]. Delay in recognition of these events and appropriate notification of care providers leads to delays in treatment and recovery, and increases morbidity and mortality risk $[9,10,14]$. Providing timely interventions requires active monitoring for early detection of health problems. This type of monitoring could be accomplished with diligent human observation, but may be viewed as undesirably intrusive [15]. Telehealth monitoring can also accomplish some of this monitoring; however, it requires that people actively 'do' something, such as take their weight, blood pressure, or other measures and submit them via telehealth equipment. While telehealth can be an effective method to manage some aspects of chronic illness, influence behavior, and improve health outcomes [16, 17], several problems persist. First, traditional telehealth equipment usually measures traditional vital signs or other common measures such as pulse oximetry, blood sugar, or weight only intermittently, typically once or twice per day [16]. Second, several studies have shown that adherence to telehealth programs decreases over time [16]. Finally, because people have to 'use' the equipment, it can be a reminder that they are not 'well' and affect perceptions of quality of life and independence.

Wearable [18], health smart homes, and home-based consumer health technologies [19] have been suggested in reviews as having much potential to support older adults as they age in place, promoting healthy lifestyles. Similarly, a technological review of monitoring health behavior and activity concluded that smart homes are suitable for older adults, as they 'may spend a large majority 
Fig. 1. Sensor system. EHR = Electronic health record.

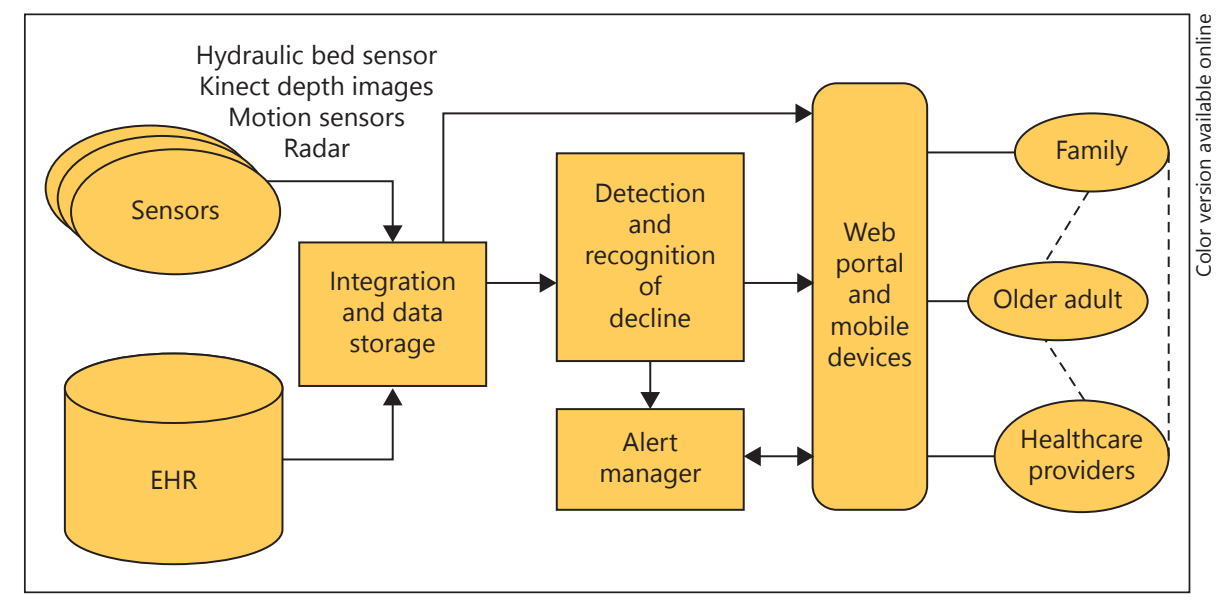

of their time in the home' [20]. A review of older adults' willingness to live with advanced technology in their homes [21] found that older adults are willing to live with technology that will improve their quality of life and help them continue to live independently.

That willingness may not extend to continuously wearing sensors for fall detection [22]. A review of sensor technologies targeting fall prevention in hospitals and other institutional settings (most frequently bed or chair alarms) concluded additional research is needed to 'develop sensor systems which cover rooms and units $24 \mathrm{~h}$ a day' and also cover larger areas, 'not only around the bed or a chair' [23, p 751]. In a review of wearable sensors for assessment of fall risk, the authors raise concerns that unsupervised assessment of fall risk in the community should not be the end goal because appropriate interventions should be planned to reduce fall risk [24]. However, since most falls of older adults go unreported, detecting an actual fall may represent the first opportunity clinicians have to offer fall risk reduction interventions [25]. Additionally, because 1 in 3 people over 65 years of age fall each year, ways of reducing fall risk are essential to the lives and well-being of large numbers of older adults [25].

\section{Sensor System Research at TigerPlace}

Clinicians in our research team envisioned new, more sensitive technological solutions could be developed to detect subtle changes in physical function or health conditions of older adults in a real setting where older adults lived that could enable interdisciplinary research. TigerPlace, named after the University of Missouri mascot, was built to act as this 'living laboratory' for conducting re- search with the targets of: (1) improving care delivery methods to older adults, (2) evaluating new interventions to improve physical and cognitive function, and (3) developing technologies to enhance aging in place [1]. The independent housing facility has 54 spacious apartments designed and regulated to allow aging in place through the end of life with the support of RN (registered nurse)led care coordination.

In 2002, during the planning and design of TigerPlace, an interdisciplinary group of researchers from the School of Nursing, College of Engineering, and other schools on the University of Missouri campus came together to begin development and testing of new technology that could enhance aging in place for older adults. Early in the planning, the team agreed that the technology developed should not require people to wear anything or actively 'do' things with the technology. This specification came from the nurses and other clinicians on the team with years of experience working with older adults; almost everyone had experienced family or patients who refused to wear 'alert pendants' or other wearable devices designed to promote assistance and safety. The challenge for the engineering team was to develop new technological options that people would be willing to use when fully developed and tested.

Since 2005, iterations of sensor systems have been developed, installed, and kept in continuous use to meet this option. The integrated sensor system ultimately developed by the team is illustrated in figure 1 and includes: (1) motion and bed sensors, (2) Microsoft Kinect, (3) pulseDoppler radar, (4) an electronic health record, (5) an integration and data storage component to house all of the data, (6) detection and recognition algorithms to determine when a resident is declining or falls, (7) an alert 
Fig. 2. Sensors in the living environment.
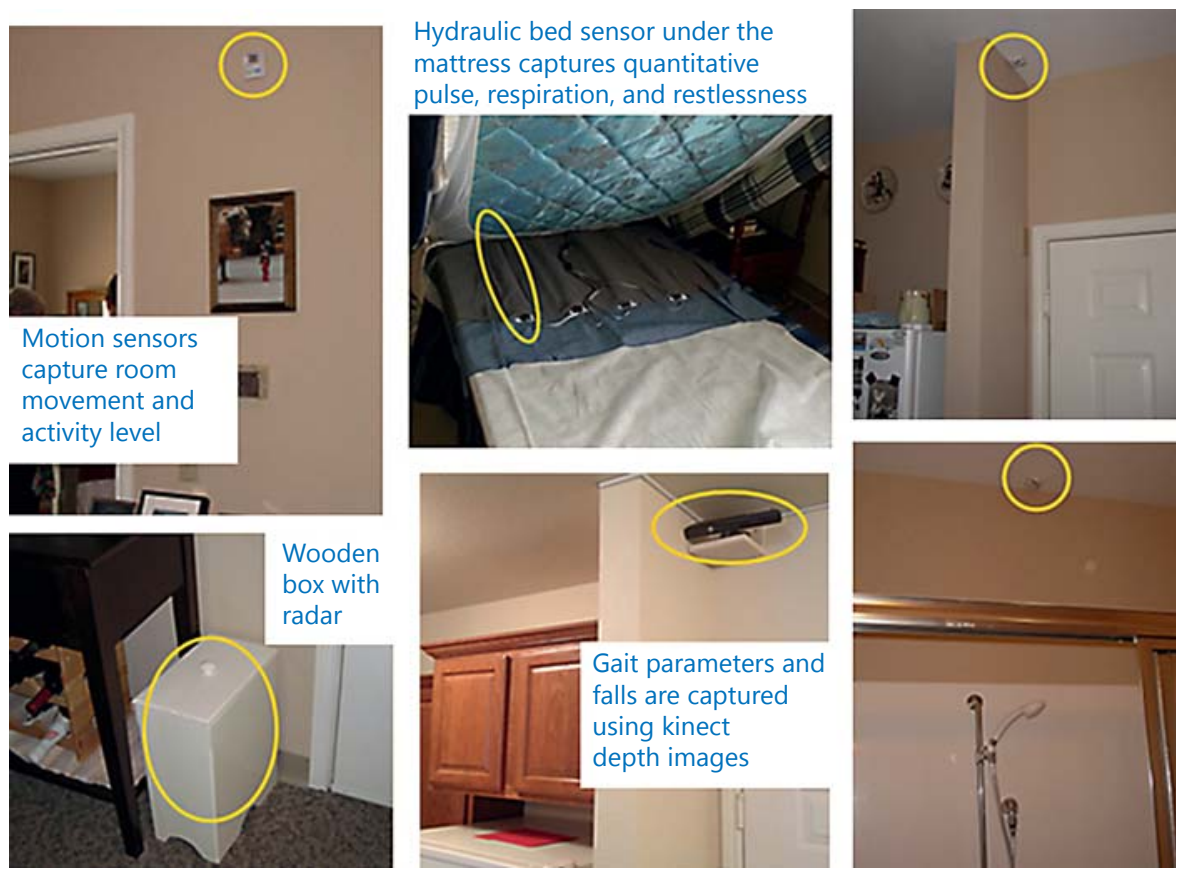

manager to automatically notify healthcare providers of potential changes in health status, and (8) a Web-based interface in which sensor data are accessible to clinicians, clients, and families, so they can interpret the data and intervene with appropriate interventions or treatment $[26,27]$.

Figure 2 shows the various sensors embedded in the environments of participating older adults living at TigerPlace. Motion sensors detect presence in a room and infer activities; for example, a sensor installed above the shower infers bathing activity. The X-10 passive infrared motion sensor was selected because it is inexpensive and unobtrusive. When our work began, clinicians and engineers discussed the frequency of X-10 sensor 'events' and concluded the 7 -second interval would provide a finely grained activity measure, but also avoid excessive battery use that would result in staff interrupting residents to change batteries. We wanted the sensor system to be as invisible as possible, with a minimum amount of hardware checks so that residents were able to live their lives with minimal interruptions. With the 7-second interval, an 'event' is generated every $7 \mathrm{~s}$ when the sensor detects continuous motion. As more motion occurs in a room, such as walking, more sensor events are generated. Fewer sensor events are generated when the resident is sedentary. These sensor events are used in displays as 'density per unit time' to help clinicians quickly infer activity level $[3,28]$. A bed sensor captures physiological parameters while sleeping. Until recently, the bed sensor used a pneumatic tube which lies on top of the mattress under the bed linens; it detected presence in bed and measured pulse, respiration, and restlessness qualitatively as high, medium, or low [29]. A more precise hydraulic bed sensor, which measures quantitative pulse, respiration, and restlessness, was recently deployed at TigerPlace [30]. All studies conducted at TigerPlace have university institutional review board approval and all participants provide written informed consent. All research papers are available on our website, http://eldertech.missouri.edu/.

\section{Sample: Sensor System Research}

Using progressive iterations of the integrated sensor system at TigerPlace, which have been in place since 2005, sensors collect data continuously, $24 \mathrm{~h}$ a day, 7 days a week. Through October 2013, 52 people (18 men, 34 women; all Caucasian; average age: 85 years, range: $63-$ $97)$ have been monitored. The average length of observation is 1.8 years. Thirty people have been discharged: 12 died, 10 moved to nursing home, 2 withdrew for personal reasons, 4 moved to assisted living, and 2 moved in with family in the community. Four couples have been monitored and the remaining participants were(are) single. Twenty-two participants remain in the monitoring program; rolling enrollment maintains $20-25$ people who are continuously monitored. 
Fig. 3. Email alert sent to clinicians for health alerts. EHR = Electronic health record.

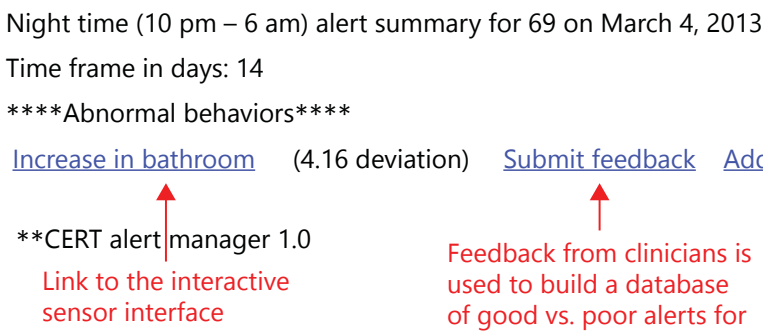

\section{Key Findings: Sensor System Research}

Similar to findings from other researchers using motion sensors, general daily activity of older adults in various locations in the living environment can be readily captured from motion sensors and interpreted by clinicians [4]. Time away from home can also be captured readily with motion sensor placement above doorways exiting the home and algorithmically confirming the absence of activity $[4,26,28]$. When more than one person lives in the home, activity can still be captured and algorithms send alerts to facility staff, family, or residents themselves to check that a change has occurred [26]. Using Kinect, walks of a resident can be accurately differentiated from those of other residents, as well as visitors, allowing his/her gait to be assessed [31]. Additionally, placement of 2 bed sensors, in cases of couples sharing beds, bed restlessness, and changes in pulse and respiration can be accurately attributed to each person.

As clinicians working on the research team became familiar with resident participants and viewed iterations of sensor data displays, they noticed changes in sensor patterns. Sometimes there were changes in activity level (increased or decreased) or in high, medium, or low pulse or respiration or restlessness while in bed or a favorite recliner where sensors were deployed. These observations led to further research, i.e. developing health alert algorithms based on the sensor data and 'new vital signs'.

\section{Discovering and Interpreting Functional Status 'Vital Signs'}

In a separate study, a systematic retrospective review of 4 years of sensor data [ $\mathrm{n}=148$ health events (falls, ER visits, hospitalizations) for 22 subjects in the sensor system research described above] was conducted with input from nursing, computer engineering, medicine, and social work [32]. This review guided initial sensor data al- gorithm development for health alerts to clinicians that signal potential changes in health status for them to evaluate. After iterative improvements in algorithms, health alerts were prospectively tested and refined in a 1-year, 2-group pilot study with 42 subjects living at TigerPlace. The intervention group $(n=20)$ lived with the sensor system in their apartments and had automated health alerts generated from their sensor data. Nursing and social work staff received alerts via email (fig. 3), assessed residents as appropriate, and intervened when necessary. The goal was to alert staff to potential changes in health status, behavior, or function facilitating early intervention and treatment, thus improving functional and health outcomes. The control group $(\mathrm{n}=22)$ received usual care provided by nursing and social work staff. Alert algorithms produce an active alert within the clinician's daily workflow that does not rely on the clinician to manually monitor activity patterns, although viewing these data are available to the clinician through a link on the alert [27].

\section{Key Findings: Health Alert Research Using the Sensor} System

Results of the 1-year pilot study revealed that the intervention group showed significant improvement as compared to the control group for the Short Physical Performance Battery [33] gait speed score at quarter 3 ( $p=$ $0.03)$, left hand grip at quarter $2(\mathrm{p}=0.02)$, right hand grip at quarter $4(\mathrm{p}=0.05)$, and GAITRite Functional Ambulation Profile score at quarter $2(p=0.05)$ [32]. These functional improvements from use of health alerts for proactive intervention by clinicians were evidence of potential early interpretation of health changes.

In addition to better functional outcomes demonstrated in the prospective study, the algorithms, on average, detect health changes 10 days to 2 weeks before the person is aware of symptoms of impending health problems [32], which they may eventually bring to the attention of staff or family. These early, subtle changes in the person's typ- 
ical patterns of activity and nighttime restlessness, pulse, and respiration are recognized by the automated algorithms. Early recognition provides much needed lead time for clinicians to intervene early when interventions are more likely to be effective without hospitalization; recovery can be faster with less functional decline and subsequent loss of independence. Common health conditions found by early assessment included acute infections such as urinary tract infections, pneumonia, upper respiratory infections, heart failure, posthospitalization pain, delirium, and hypoglycemia [32]. Based on these results, a larger randomized trial is underway with 140 older adults in assisted and independent living environments throughout mid-Missouri.

Mental health and cognitive changes can also be detected by the sensor systems. Clinicians first noticed changes in activity levels, time out of the apartment, and time in bed, which they suspected might be linked to depression or onset or progression of dementia. Again, a retrospective review of sensor data was conducted for all residents who participated in the sensor system research with a mental health diagnosis of dementia or depression. Patterns in activity, sleep, and time away from home revealed patterns of change that coincided with clinical changes recorded in health assessments in the clinical record [34]. Interventions to improve depression or better manage the progression of dementia improved symptoms for most, but not all, subjects. The one resident who did not show improvement had a chronic condition characterized by steady declines in functional ability. In this case, the resident experienced longer periods of stability within a trajectory of overall decline. Today, clinicians at TigerPlace receive real-time alerts to potential mental health changes. They complete more in-depth assessments and implement or refer for interventions/treatments, which, in turn, may eliminate or decrease the severity of depression or delay progression of dementia [27].

\section{Detecting and Assessing Fall Risk and Fall Occurrences}

In a pair of separate studies, a fall detection and fall risk assessment (FDFRA) system was developed and continuously used in 10 TigerPlace apartments for 2 years as part of the sensor system. The FDFRA system has tested range controlled pulse-Doppler radar (developed by GE Global Research), Microsoft Kinect (originally developed as part of a gaming system), and two web cameras. The radar unit is installed in a decorative wooden box by the front door, and Kinect is located on a small wooden shelf above the front door (fig. 2). The web cameras are installed on orthogonal walls of the living room. To preserve the privacy of residents, only signals from the radar and the 'silhouette-like' Kinect depth image (where each pixel contains information about the distance from the camera) [35] were continuously stored. The foreground silhouette images from the web cameras were stored only during monthly fall risk assessment (FRA) data collection [36, 37].

The FDFRA system was first tested in the engineering research laboratory at the University of Missouri where the Vicon motion capture system was used as 'ground truth' for validation of radar, Kinect, and other sensing modalities [38]. The Vicon system uses reflective markers worn by subjects and a system of cameras to precisely measure torso and limb movements. Fifteen adult volunteers (average age: 56 years, range: 23-67) performed a FRA protocol of 6 standardized fall risk measures used by healthcare providers, all with acceptable validity and reliability [36], as the sensors and Vicon simultaneously collected data. Similarly, two trained stunt actors performed a series of falls to provide data for the initial development of the fall detection algorithms for the sensor data.

\section{Sample: FDFRA System Research}

Twenty-two subjects (10 men, 12 women; all Caucasian) ages 67-98 years ( 3 couples, 16 single participants) have been monitored with the FDFRA system while living at TigerPlace. More than 2 years of continuous data have been collected, and the average length of monitoring per subject is 1.75 years. Although there are challenges monitoring more than one person in the environment, we have developed algorithms for FDFRA to differentiate walking of the primary people who live in the environment [31]. Each month, for 2 years, participants performed the FRA protocol of 6 standardized measures in their apartments and a stunt actor performed a protocol of falls while all the sensors recorded their movements. These FRAs and actual falls were used to further develop and refine algorithms for FRA and fall detection. The number of FRAs for each subject has varied based on length of participation.

As the research progressed, naturally occurring falls enhanced the data for algorithm refinement. Although the stunt actor falls are good, many of the actual falls by seniors differ somewhat from stunt actors. Residents often fall more slowly, and they are often holding on to a walker, or furniture, during the fall. Adding these to the 
Fig. 4. Email fall alert.

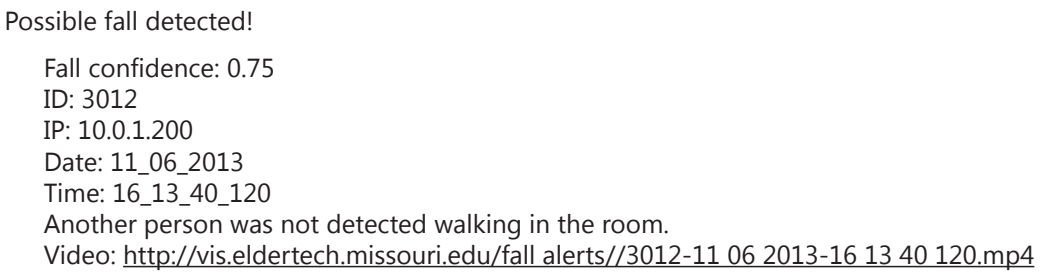

training data for the algorithm improved the classifiers since there are no representative samples of actual older adult falls from stunt actor data.

\section{Key Findings: Fall Risk and Fall Detection Research Using FDFRA}

Algorithms for continuous in-home FRA were developed to accurately extract gait characteristics of mean velocity, stride duration, and stride variability from the radar signal $[39,40]$. In addition, a fall detection algorithm was developed to detect actual falls from radar signals [41]. More recently, radar units were installed in the ceiling over the living room and bathroom area of participants' apartments. Radar units located in ceilings have shown better results in detecting actual falls than those deployed near the floor [42].

Algorithms for automated continuous FRA were developed for Kinect. From Kinect data, gait velocity, stride length, and stride time are automatically calculated [31, 35]. Height is computed from depth data aiding in differentiation of individuals when multiple people live or visit in the same apartment. The FRAs collected monthly in participants' apartments provided 'ground truth' for development of a new automated fall risk measure, average in-home gait speed. In an analysis of 203 paired observations of the 6 standardized FRAs and Kinect data from the same day for 18 subjects (described above), most Kinect measurements were significantly correlated with all of the FRAs $(\mathrm{p}<0.01)$ [43]. It may be likely that average inhome gait speed is better suited for detecting subtle changes in an individual's fall risk over time than traditional fall risk measures conducted sporadically by physical therapists or other clinical staff [44]. Whereas traditional measures provide a snapshot of physical function, average in-home gait speed is a continuous measure computed using normal everyday activity with privacy-protecting depth images.

The Kinect fall detection system sends an alert via email to clinical staff when a participant falls (fig. 4). Embedded in the 'real-time' alert is a link for clinical staff to

Technology-Enabled 'Vital Signs'

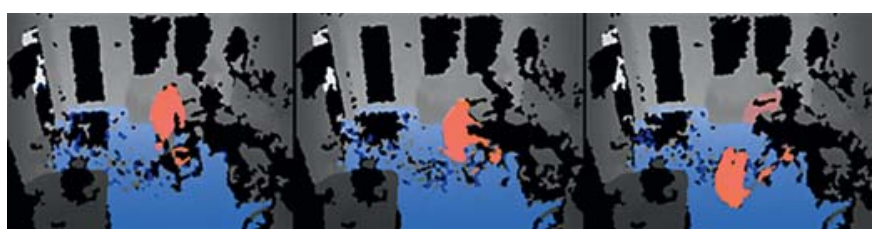

Fig. 5. Depth images of a TigerPlace resident fall detected by Kinect.

'click' so they can immediately view the 'silhouette-like' images from several seconds before, during, and after the event that the automated algorithm detected as a fall (fig. 5). Clinical staff view these alerts and linked images from a smart phone or other email-viewing device so they can determine quickly if an actual fall occurred. This process speeds response time for an actual fall event, while avoiding unnecessary staff intrusion for a nonfall event (such as a pillow tossed on the floor). Use of research ID numbers and silhouette images allow review of the event while preserving resident privacy, an approach the residents found acceptable [37, 45]. Importantly, depth images can be reviewed after a fall to examine individual and environmental factors that may have triggered the fall. Intervention may then be implemented to address fall risk factors and subsequently reduce risk of future falls. The real-time Kinect fall detection system was implemented at TigerPlace in August 2013, and staff and residents provided positive feedback on the system with few suggestions for change. In late 2013, the research team and clinicians decided that Kinect would be the primary FRA and fall sensor used at TigerPlace and other sites as it is more robust than a web camera or radar. Web cameras and floor radar were removed from TigerPlace in 2014.

In addition to deployment of Kinect in home environments [46], the research team is pilot testing Kinect for fall detection in a hospital [47]. While falls are a common occurrence in hospitals, they are rarely seen by staff as they occur. This lack of contextual information can frustrate clinical staff to accurately discern the 'root cause' of 
fall occurrences as well as determine what injuries were and were not likely to have occurred. Currently, pilot testing is expanding to other patient units and a second hospital site in Missouri.

\section{Obstacles to Overcome for Mainstream Adoption and} Use

Several obstacles remain for mainstream adoption of a health alert system using the new 'vital signs' of change in activity as well as changes in bed restlessness, pulse, or respirations. A larger sample in a more diverse population of older adults is needed to validate results and ensure they are replicable in a variety of settings. Additional usability studies of displays of sensor information and alerts with not only clinicians but also older adults and their family members are needed to assure displays are easy to use and understand. A larger intervention study is currently underway to validate results and test the system in 12 assisted and independent living facilities. This larger study will provide a more diverse set of clinicians as well as older adults and their family members who will use the displays.

For the FDFRA system, the most robust automated FDFRA sensor we have located and studied is Kinect. While we have been doing real-time fall detection in both TigerPlace and a hospital setting, more work is needed. For example, Kinect can only detect falls that occur within range of the sensor. When there are occlusions in the field, such as furniture, the sensor is often unable to detect falls. Some of this can be overcome with the proper placement of the sensor, but some falls will be missed if they occur outside of the sensor's field of view.

From the perspective of privacy and preferences, continuing studies with consumers are needed. Thus far, Kinect sensors are installed in the living rooms of participants. No Kinect sensors have been installed in bedrooms or bathrooms because of privacy concerns. These concerns need to be addressed before the sensor can be widely deployed. While we have always included interviews of participants living with the technology about their perceptions and willingness to live with technology [45, 48], more diverse populations of consumers are needed to be sure that all privacy concerns are met and that preferences in the use of technology are honored with varying groups of older adult participants. Based on our experience, research, and findings of the most recent review of older adults' willingness to live with technology [21], we believe they are willing as long as the technology is unobtrusive, helps them feel safer living in their home, they control who sees the data, and the technology meets a perceived need.

\section{New Paradigm of Technology-Enhanced 'Vital Signs'}

A new paradigm of healthcare monitoring is emerging at TigerPlace, without people wearing devices or actively 'doing things'. Older adult participants' respiration, pulse, and restlessness are monitored as they sleep. Gait speed, stride time, and stride length are calculated daily. Activity levels are continuously summarized and graphically displayed for easy interpretation. All are easily accessed through a Web-based, privacy protecting interface. Automated health alerts are sent to healthcare staff, based on validated algorithms from the sensor data, days and weeks before typical signs or symptoms are detected by the person, family members, or healthcare providers. Falls are detected and alerts sent immediately to healthcare providers so that time to rescue and treatment can be reduced. Technology-enhanced 'vital signs' provide a more comprehensive picture of a person's health and functional status than traditional vital signs. This paradigm has the potential to transform the way chronic disease is managed and can help older adults age in place with less fear of undetected debilitating illnesses or falls.

We began by introducing Mary, who had recently become tired, less active, and had fallen. Consider Mary's situation with embedded environmental sensors:

Louise used to check on Mary, her 86-year-old mother, daily by phone, but now calls her merely to chat about her day, as she receives alerts by email if anything is out of the ordinary. Louise is somewhat concerned today because she has been receiving alerts for the last 2 days that her mother is not resting well and that she did not leave the apartment today despite it being the day for her bridge club. She also got an alert yesterday that her mother used the bathroom much more than usual. Louise logs into the sensor system and notes that her mother seems to be spending a lot of time in the living room, probably in her favorite chair, but that overall she is not very active. There is also no activity recorded in the kitchen in the last $24 \mathrm{~h}$, causing Louise to be concerned that Mary is not eating. Louise calls her mother's home care nurse, who is scheduled to visit Mary today, to relay her concerns. Later that day, the nurse calls Louise and lets her know that based on the history of extra bathroom visits, they checked her mother's urine and it showed both high glucose and signs of infection. They shared this information with Mary's doctor who will see her later today. Louise takes off work to accompany her mother to the doctor, where she is prescribed treatment for a urinary tract infection. They also find that Mary's blood sugar is slightly elevated due to the infection, but not dangerously so. They make a plan to check her blood sugar more frequently over the next few days. Louise, concerned that her mother has little appetite, decides to stay overnight with Mary. She encourages her mother to drink and eat, and the next day Mary is feeling a little more like herself. Early detection led to early intervention and Mary did not experience a fall. Moreover, her illness did not progress to a more serious infection requiring a hospitalization... . 


\section{Acknowledgement}

This article is based on results of several funded research projects including, NINR funded research, 1R21NR011197-02 (Rantz, PI, 2009-2012); US National Science Foundation grants CNS-0931607, IIS-0428420, IIS-0703692, and CNS-1237970 (Skubic, PI); IIS-1115956 (Popescu, PI); Agency for Healthcare Research and Quality under grant R01-HS018477 (Rantz, PI);
NIH NINR 1R01NR014255 (Rantz, PI), and US Administration on Aging, grant No. 90AM3013 (Rantz, PI). Results and conclusions are the responsibility of the researchers, and not the opinions of funding agencies. Authors gratefully acknowledge the residents and staff of TigerPlace who graciously participate in our technology development research and the students and faculty of the Eldertech Research Team at the University of Missouri.

\section{References}

1 Rantz MJ, Marek KD, Aud MA, Tyrer HW, Skubic M, Demiris G, Hussam AA: A technology and nursing collaboration to help older adults age in place. Nurs Outlook 2005;53:40-45.

-2 Alexander GL, Rantz M, Skubic M, Aud MA, Wakefiled B, Florea E, Paul A: Sensor systems for monitoring functional status in assisted living facility residents. Res Gerontol Nurs 2008;1:238-244.

3 Alexander GL, Rantz MJ, Skubic M, Koopman R, Phillips L, Guevara RD, Miller S: Evolution of an early illness warning system to monitor frail elders in independent living. J Healthc Eng 2011;2:259-286.

4 Rantz MJ, Skubic M, Alexander G, Aud M, Wakefield B, Koopman R, Miller S: Improving nurse care coordination with technology. Comput Inform Nurs 2010;28:325-332.

5 Ward BW, Schiller JS, Goodman RA: Multiple chronic conditions among US adults: a 2012 update. Prev Chronic Dis 2014;11:130389.

6 Chronic Care: Making the Case for Ongoing Care. Princeton, Robert Wood Johnson Foundation, 2010, p 16. http://www.rwjf.org/ content/dam/farm/reports/reports/2010/ rwjf54583.

7 Centers for Disease Control and Prevention. Improving the health of older Americans: a CDC priority. Chronic Dis Notes Rep 2007;18: 3-6. http://www.cdc.gov/aging/pdf/CDNR. June.2007.pdf.

8 'What is chronic disease?' http://cmcd.sph. umich.edu/what-is-chronic-disease.html (accessed February 23, 2014).

-9 Boockvar KS, Lachs MS: Predictive value of nonspecific symptoms for acute illness in nursing home residents. J Am Geriatr Soc 2003;51:1111-1115.

10 Ridley S: The recognition and early management of critical illness. Ann R Coll Surg Engl 2005;87:315-322.

-11 Fried LP, Bandeen-Roche K, Chaves PH, Johnson BA: Preclinical mobility disability predicts incident mobility disability in older women. J Gerontol A Biol Sci Med Sci 2000; 55:M43-M52.

12 Onder G, Penninx BW, Ferrucci L, Fried LP, Guralnik JM, Pahor M: Measures of physical performance and risk for progressive and catastrophic disability: results from the Women's Health and Aging Study. J Gerontol A Biol Sci Med Sci 2005;60:74-79.
13 Wolinsky FD, Miller DK, Andresen EM, Malmstrom TK, Miller JP: Further evidence for the importance of subclinical functional limitation and subclinical disability assessment in gerontology and geriatrics. J Gerontol B Psychol Soc Sci 2005; 60:S146-S151.

14 Soler-Cataluna JJ, Martinez-Garcia MA, Roman Sanchez P, Salcedo E, Navarro M, Ochando R: Severe acute exacerbations and mortality in patients with chronic obstructive pulmonary disease. Thorax 2005;60:925932.

15 Demiris G, Rantz M, Aud M, Marek K, Tyrer H, Skubic M, Hussam A: Older adults' attitudes towards and perceptions of 'smart home' technologies: a pilot study. Med Inform Internet Med 2004;29:87-94.

16 Paré G, Jaana M, Sicotte C: Systematic review of home telemonitoring for chronic diseases: the evidence base. J Am Med Inform Assoc 2007;14:269-277.

17 Gaikwad R, Warren J: The role of homebased information and communications technology interventions in chronic disease management: a systematic literature review. Health Informatics J 2009;15:122146.

18 Naranjo-Hernandez D, Roa LM, Reina-Tosina J, Estudillo-Valderrama MA: SoM: a smart sensor for human activity monitoring and assisted healthy ageing. IEEE Trans Biomed Eng 2012;59:3177-3184.

19 Reeder B, Meyer E, Lazar A, Chaudhuri S, Thompson HJ, Demiris G: Framing the evidence for health smart homes and homebased consumer health technologies as a public health intervention for independent aging: a systemic review. Int J Med Inform 2013;82: 565-579.

20 Lowe SA, Olaighin G: Monitoring human health behavior in one's living environment: a technological review. Med Eng Phys 2014; 36:147-168.

21 Jacelon CS, Hanson A: Older adults' participation in the development of smart environments: an integrated review of the literature. Geriatr Nurs 2013;34:116-121.

22 Gietzelt M, Spehr J, Ehmen Y, Wegel S, Feldwieser F, Meis M, Marschollek M, Wolf KH, Steinhagen-Thiessen E, Gövercin M: GAL@ Home: a feasibility study of sensor- based in-home fall detection. Z Gerontol Geriatr 2012;45:716-721.

23 Kosse NM, Brands K, Bauer JM, Hortobagyi T, Lamoth CJ: Sensor technologies aiming at fall prevention in institutionalized old adults: a synthesis of current knowledge. Int J Med Inform 2013;82:743-752.

24 Shany T, Redmond SJ, Marschollek M, Novell $\mathrm{NH}$ : Assessing fall risk using wearable sensors: a practical discussion. A review of the practicalities and challenges associated with the use of wearable sensors for quantification of fall risk in older people. Z Gerontol Geriatr 2012;45:694-706.

25 Martin FC: Next steps for falls and fracture reduction. Age Ageing 2009;38:640-643.

26 Skubic M, Alexander G, Popescu M, Rantz M, Keller J: A smart home application to eldercare: current status and lessons learned. Technol Health Care 2009; 17:183-201.

-27 Rantz MJ, Skubic M, Miller SJ, Galambos C Alexander G, Keller J, Popescu M: Sensor technology to support aging in place. J Am Med Dir Assoc 2013;14:386-391.

28 Wang S, Skubic M, Zhu Y: Activity density map visualization and dissimilarity comparison for eldercare monitoring. IEEE Trans Inf Technol Biomed 2012;16:607-614.

29 Mack DC, Patrie JT, Suratt PM, Felder RA, Alwan M: Development and preliminary validation of heart rate and breathing rate detection using a passive, ballistocardiography-based sleep monitoring system. IEEE Trans Inf Technol Biomed 2009;13:111120.

30 Heise D, Rosales L, Sheahen M, Su BY, Skubic M: Non-invasive measurement of heartbeat with a hydraulic bed sensor: progress, challenges, and opportunities. Proceedings 2013 IEEE International Instrumentation and Measurement Technical Conference, Minneapolis, 2013, pp 4356-4360.

31 Stone EE, Skubic M: Unobtrusive, continuous, in-home gait measurement using the $\mathrm{Mi}$ crosoft Kinect. IEEE Trans Biomed Eng 2013; 60:2925-2932.

32 Rantz MJ, Skubic M, Koopman RJ, Alexander G, Phillips L, Musterman KI, Back JR, Aud MA, Galambos C, Guevara RD, Miller SJ: Automated technology to speed recognition of signs of illness in older adults. J Gerontol Nurs 2012;38:18-23. 
33 Guralnik JM, Simonsick EM, Ferrucci L, Glynn RJ, Berkman LF, Blazer DG, et al: A short physical performance battery assessing lower extremity function: association with self-reported disability and prediction of mortality and nursing home admission. J Gerontol 1994;49:M85-M94.

34 Galambos C, Skubic M, Wang S, Rantz M: Management of dementia and depression utilizing in-home passive sensor data. Gerontechnology 2013;11:457-468.

35 Stone E, Skubic M: Evaluation of an inexpensive depth camera for in-home gait assessment. J Ambient Intell Smart Environ 2011;3: 349-361.

-36 Anderson D, Luke RH, Keller JM, Skubic M, Rantz M, Aud M: Modeling human activity from voxel person using fuzzy logic. IEEE Trans Fuzzy Syst 2009;17:39-49.

- 37 Demiris G, Parker-Oliver D, Giger J, Skubic M, Rantz M: Older adults' privacy considerations for vision based recognition methods of eldercare applications. Technol Health Care 2009;17:41-48.

38 Wang F, Stone E, Skubic M, Keller J, Abbott C, Rantz M: Towards a passive low-cost inhome gait assessment system for older adults. IEEE J Biomed Health Inform 2013;17:346355.
39 Yardibi T, Cuddihy P, Genc S, Bufi C, Skubic M, Rantz M, Liu L, Phillips C: Gait characterization via pulse-Doppler radar. Proceedings 9th Annual IEEE International Conference on Pervasive Computing and Communications: Workshop on Smart Environments to Enhance Health Care, Seattle, 2011, pp 662 667.

40 Cuddihy PE, Yardibi T, Legenzoff ZJ, Liu L, Phillips CE, Abbott C, Galambos C, Keller J, Popescu M, Back J, Skubic M, Rantz MJ: Radar walking speed measurements of seniors in their apartments: technology for fall prevention. Proceedings 34th Annual International Conference of the IEEE Engineering in Medicine and Biology Society, San Diego, 2012, pp 260-263.

41 Liu L, Popescu M, Rantz M, Skubic M: Fall detection using Doppler radar and classifier fusion. Proceedings International Conference on Biomedical and Health Informatics (BHI 2012) Hong Kong, 2012, pp 180-183.

42 Liu L, Popescu M, Ho KC, Skubic M, Rantz M: Doppler radar sensor positioning in a fall detection system. Proceedings 34th Annual International Conference of the IEEE Engineering in Medicine and Biology Society (EMBC'12), San Diego, 2012, pp 256-259.
43 Rantz MJ, Skubic M, Abbott, C, Galambos C, Popescu M, Keller J, Stone E, Back J, Miller SJ, Petroski G: An automated in-home fall risk assessment and detection sensor system for older adults. Submitted to Gerontologist, July 2014, in review.

44 Stone E, Skubic M, Rantz MJ, Abbott C, Miller S: Average in-home gait speed: investigation of a new metric for mobility and fall risk assessment of elders. Gait Posture 2014, Epub ahead of print.

45 Courtney KL, Demiris G, Rantz MJ, Skubic M: Needing smart home technologies: the perspectives of older adults in continuing care retirement communities. Inform Prim Care 2008; 16:195-201.

46 Stone E, Skubic M: Fall detection in homes of older adults using the Microsoft Kinect. IEEE J Biomed Health Inform 2014, Epub ahead of print.

47 Rantz M, Banerjee TS, Cattoor E, Scott SD, Skubic M, Popescu M: Automated fall detection with quality improvement 'rewind' to reduce falls in hospital rooms. J Gerontol Nurs 2014;40:13-17.

48 Demiris G, Hensel BK, Skubic M, Rantz MJ: Senior residents' perceived need of and preferences for 'smart home' sensor technologies. Int J Technol Assess Health Care 2008;24:120-124. 